\title{
Detection of Spin Entanglement via Spin-Charge Separation in Crossed Tomonaga-Luttinger Liquids
}

\author{
Alexander Schroer, ${ }^{1}$ Bernd Braunecker, ${ }^{2}$ Alfredo Levy Yeyati, ${ }^{3}$ and Patrik Recher ${ }^{1,4}$ \\ ${ }^{1}$ Institut für Mathematische Physik, Technische Universität Braunschweig, D-38106 Braunschweig, Germany \\ ${ }^{2}$ Scottish Universities Physics Alliance, School of Physics and Astronomy, University of St Andrews, \\ North Haugh, St Andrews KY16 9SS, United Kingdom \\ ${ }^{3}$ Departamento de Física Teórica de la Materia Condensada, Condensed Matter Physics Center (IFIMAC), \\ and Instituto Nicolás Cabrera, Universidad Autónoma de Madrid, E-28049 Madrid, Spain \\ ${ }^{4}$ Interactive Research Center of Science, Tokyo Institute of Technology, 2-12-1 Ookayama, Meguro, Tokyo 152-8551, Japan
}

(Received 17 April 2014; revised manuscript received 9 November 2014; published 23 December 2014)

\begin{abstract}
We investigate tunneling between two spinful Tomonaga-Luttinger liquids (TLLs) realized, e.g., as two crossed nanowires or quantum Hall edge states. When injecting into each TLL one electron of opposite spin, the dc current measured after the crossing differs for singlet, triplet, or product states. This is a striking new non-Fermi liquid feature because the (mean) current in a noninteracting beam splitter is insensitive to spin entanglement. It can be understood in terms of collective excitations subject to spin-charge separation. This behavior may offer an easier alternative to traditional entanglement detection schemes based on current noise, which we show to be suppressed by the interactions.
\end{abstract}

Entanglement is a necessary prerequisite for universal quantum computation and certain quantum communication protocols like quantum teleportation or dense coding [1]. The creation of nonlocal pairwise entangled particles has been successfully demonstrated with photons [2-4] by violating a Bell inequality $[5,6]$. The same has not yet been demonstrated in transport experiments in a solid state device. In particular, spin-entangled electrons are important candidates, because the electron spin in quantum dots could be used as a qubit [7], with proven promising spin-coherence times [8]. From the theoretical side, Cooper-pair splitters (CPSs) [9-16] were proposed as a potential source of mobile and nonlocal spin-entangled pairs, using the process of crossed Andreev reflection [17,18]. Experimentally, such CPSs have been built successfully [19-21] with high efficiency [22]. However, the spin entanglement of these correlated pairs has not been demonstrated so far. Several detection schemes for entangled states were proposed based on a violation of a Bell inequality using cross-correlation (noise) measurements [23-29], current measurements in a CPS with spin-filter properties [30], or exploiting beam splitters [31-39] where a bunching or an antibunching behavior in the two-electron scattering process depends on the orbital wave function of the entangled pairs, distinguishing singlets from triplets or product states. The latter is an effect of statistics and holds already for noninteracting electrons. The average current does not carry a signature of entanglement in Fermi-liquid systems [31].

In this Letter we show that the situation is radically different in the case of a beam splitter made of one-dimensional interacting nanowires $[40,41]$, or, almost equivalently, integer quantum Hall $(\mathrm{QH})$ edge states [42-46]. In these systems, which can conveniently be described as TomonagaLuttinger liquids (TLLs) [47,48], the average current is sensitive to spin entanglement due to the property of spincharge separation. This is a desirable feature because the current is generally much easier to measure than noise or higher-order correlation functions. An interpretation of the current noise in terms of (anti-)bunching [31] still applies, although Coulomb repulsion reduces the signal. The TLL system allows for an entangler $[11,12,16]$ and detector scheme without the need of magnetic elements (spin filters) nor noise-correlation measurements. Experimentally, transport through crossed one-dimensional conductors has already been demonstrated, including TLL effects [49-52]. We focus on the slightly more general case of nanowires and give details about a $\mathrm{QH}$ implementation in the Supplemental Material [53].

Model.-We consider two long nanowires (wire 1 and wire 2), which are connected through a weak tunnel junction at $x=0$ (Fig. 1). To the left of the junction, at $x_{1}, x_{2}<0$, electrons are injected pairwise from an entangler, biased with a voltage $V$. The temperature is assumed smaller than the bias voltage and can be set to zero for convenience. The rate of injection is sufficiently low that there are no correlations between subsequent electron pairs.

In the TLL left- and right-moving electron modes are expressed as bosonic fluctuations described by the Hamiltonian [54-56]

$$
H_{0}=\sum_{j \alpha} \int d x \frac{\hbar v_{\alpha}}{2}\left[g_{\alpha}\left(\frac{\partial \phi_{j \alpha}}{\partial x}\right)^{2}+\frac{1}{g_{\alpha}}\left(\frac{\partial \theta_{j \alpha}}{\partial x}\right)^{2}\right],
$$

with $\phi$ and $\theta$ dual phase fields obeying $\left[\theta_{j \alpha}(x, t)\right.$, $\left.\phi_{j^{\prime} \alpha^{\prime}}\left(x^{\prime}, t\right)\right]=(i / 2) \delta_{j j^{\prime}} \delta_{\alpha \alpha^{\prime}} \operatorname{sgn}\left(x-x^{\prime}\right)$. In this notation, 


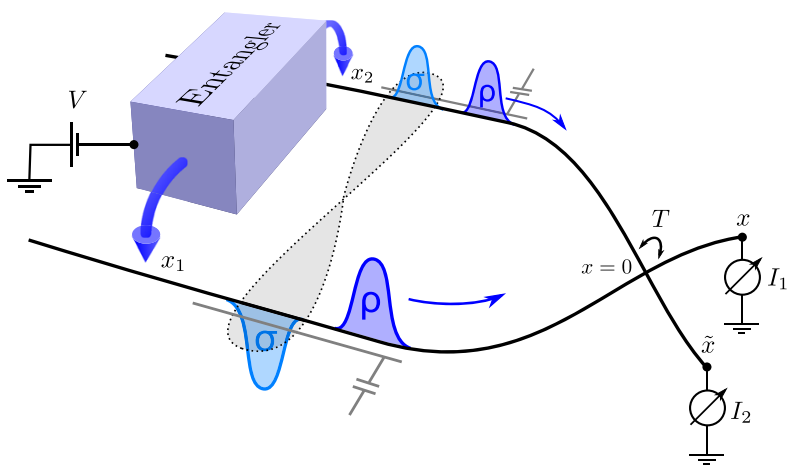

FIG. 1 (color online). Tunnel junction with amplitude $T$ at $x=0$ between two interacting one-dimensional wires. Via an entangler biased with a voltage $V$, two spin-entangled electrons are injected simultaneously at $x_{1}$ in wire 1 and at $x_{2}$ in wire 2 with an amplitude $I$, and subsequently decay into collective spin and charge excitations. The current expectation values $I_{1,2}$ measured at $x, \tilde{x}$ at the far opposite side of the junction and their cross correlations are influenced by the entanglement of the original electrons.

$\partial_{x} \phi_{j \alpha}$ is proportional to the charge current $(\alpha=\rho)$ or the spin current $(\alpha=\sigma)$ in wire $j \in\{1,2\}$ and $\partial_{x} \theta_{j \alpha}$ to the corresponding density. Assuming SU(2) spin invariance, the interaction parameter in the spin sector is $g_{\sigma}=1$, and in the charge sector, $g_{\rho} \equiv g<1$. With $v_{F}$ the Fermi velocity, $v_{\rho}=v_{F} / g_{\rho}$ and $v_{\sigma}=v_{F}$ are the velocities of spin and charge excitations. The physical electron field with spin $s \in\{\uparrow, \downarrow\}$ is $\psi_{j s}=\psi_{j R s}+\psi_{j L s}$ with the right $(R)$ - and left $(L)$-moving contribution $\psi_{j R / L s}(x, t)=$ $(2 \pi a)^{-1 / 2} F_{j R / L s} \exp \left[ \pm i k_{F} x+2 \pi i \Phi_{j R / L s}(x, t)\right]$ and $\Phi$ a linear combination of $\phi$ and $\theta[53,55,56]$. The Klein factors $F$ are unitary, anticommuting operators ensuring fermionic commutation relations [55]. The cutoff parameter of the wires, $a$, corresponds to their inverse bandwidth.

The tunnel junction at $x=0$ is described by the Hamiltonian

$$
H_{T}=T \sum_{\substack{\left.\nu, \nu^{\prime} \in\{R, L\} \\ s \in\{\uparrow,\}^{\prime}\right\}}}\left(\psi_{1 \nu^{\prime} s}^{\dagger}(0) \psi_{2 \nu s}(0)+\psi_{2 \nu^{\prime} s}^{\dagger}(0) \psi_{1 \nu s}(0)\right) .
$$

Initial state approach.-First, we include the entangled electron pair as a suitably chosen initial state at time $t_{0}$ with one electron in each wire at $x_{1}$ and $x_{2}$, respectively, on top of the many-particle ground state |\rangle

$$
\begin{aligned}
|\varphi\rangle & =\frac{\pi a}{\sqrt{2}}\left(\psi_{2 \downarrow}^{\dagger}\left(x_{2}\right) \psi_{1 \uparrow}^{\dagger}\left(x_{1}\right)+e^{i \varphi} \psi_{2 \uparrow}^{\dagger}\left(x_{2}\right) \psi_{1 \downarrow}^{\dagger}\left(x_{1}\right)\right)|\rangle \\
& :=2^{-1 / 2} \sum_{\nu_{1}, \nu_{2}}\left(\left|\nu_{1} \uparrow, \nu_{2} \downarrow\right\rangle+e^{i \varphi}\left|\nu_{1} \downarrow, \nu_{2} \uparrow\right\rangle\right) .
\end{aligned}
$$

The relative phase $\varphi$ is the rotation angle between the pure triplet state $(\varphi=0)$ and the pure singlet state $(\varphi=\pi)$. We choose $\left|x_{1,2}\right| \gg a$ to avoid initial overlap between the injected electrons and the tunnel contact. Later, we will show that the results of this model carry over to the case of an applied bias voltage $V$ by essentially replacing the wave-packet width $a$ of the state $|\varphi\rangle$ by $\hbar v_{F} / e V$, where $e$ is the electron charge.

Every expectation value of an operator $\mathcal{O}$ with respect to these states can be written as $\langle\varphi|\mathcal{O}| \varphi\rangle=$ $\mathcal{O}^{\text {dir }}+\cos (\varphi) \mathcal{O}^{\text {exc }}$, where the direct term $\mathcal{O}^{\text {dir }}=$ $\sum_{\nu_{1} \nu_{2}}\left\langle\nu_{1} \uparrow, \nu_{2} \downarrow|\mathcal{O}| \nu_{1} \uparrow, \nu_{2} \downarrow\right\rangle$ is the product state contribution, and the exchange term $\mathcal{O}^{\text {exc }}=\sum_{\nu_{1} \nu_{2}}\left\langle\nu_{1} \uparrow, \nu_{2} \downarrow|\mathcal{O}| \nu_{1} \downarrow, \nu_{2} \uparrow\right\rangle$ is a distinctive indicator of entanglement [31]. Varying $\varphi$ is a powerful way to identify the exchange contribution in a measurement, which will be discussed later on.

Within this approach, the current expectation value in wire 1 after the injection is given by

$$
I_{1}=e \Gamma_{2 e} \int_{t_{0}}^{\infty} d t\left\langle\varphi\left|I_{1}(x, t)\right| \varphi\right\rangle
$$

with $x \gg a, \Gamma_{2 e} \ll v_{F} a^{-1}$ the rate of injection, and the bosonized current operator [56] $I_{j}(x, t)=-\sqrt{2 / \pi} \partial_{t} \theta_{j}(x, t)$. Similarly, the zero-frequency cross-correlations between the two wires are

$$
S_{12}=\frac{e^{2} \Gamma_{2 e}}{2} \int_{t_{0}}^{\infty} d t d t^{\prime}\left\langle\varphi\left|\left\{\delta I_{1}(x, t), \delta I_{2}\left(\tilde{x}, t^{\prime}\right)\right\}\right| \varphi\right\rangle,
$$

where $\delta I_{j}=I_{j}-\left\langle\varphi\left|I_{j}\right| \varphi\right\rangle$.

Treating $H_{T}$ as a perturbation [57], the expressions Eqs. (4) and (5) can be evaluated with a standard Keldysh nonequilibrium generating functional approach [58-60]. Besides the zeroth-order contributions (no tunnel processes) $I_{1}^{(0)}=-e\left(\Gamma_{2 e} / 2\right)$ and $S_{12}^{(0)}=0$ they yield second order in $T$ direct and exchange corrections. The former contain effects due to interactions and spin-charge separation, which are further discussed in the Supplemental Material [53], but they are not sensitive to entanglement. The latter are

$$
\begin{aligned}
I_{1}^{(2) \mathrm{exc}}= & e \Gamma_{2 e} \frac{1+g}{2} \\
& \times\left[\left\langle R \uparrow, R \downarrow\left|U_{1 R \rightarrow 2 R}^{(1) \uparrow \dagger} U_{1 R \rightarrow 2 R}^{(1) \downarrow}\right| R \downarrow, R \uparrow\right\rangle\right. \\
& \left.-\left\langle R \uparrow, R \downarrow\left|U_{2 R \rightarrow 1 R}^{(1) \downarrow \dagger} U_{2 R \rightarrow 1 R}^{(1) \uparrow}\right| R \downarrow, R \uparrow\right\rangle\right], \\
S_{12}^{(2) \text { exc }}= & -e^{2} \Gamma_{2 e}\left(\frac{1+g}{2}\right)^{2} \\
& \times \operatorname{Re}\left[\left\langle R \uparrow, R \downarrow\left|U_{1 R \rightarrow 2 R}^{(1) \uparrow \dagger} U_{1 R \rightarrow 2 R}^{(1) \downarrow}\right| R \downarrow, R \uparrow\right\rangle\right. \\
& \left.+\left\langle R \uparrow, R \downarrow\left|U_{2 R \rightarrow 1 R}^{(1) \downarrow \dagger} U_{2 R \rightarrow 1 R}^{(1) \uparrow}\right| R \downarrow, R \uparrow\right\rangle\right] .
\end{aligned}
$$

Here, $U_{j R \rightarrow k R}^{(1) s}=-\left.i \hbar^{-1} \int_{t_{0}}^{\infty} d t^{\prime} H_{\mathrm{T}}\left(t^{\prime}\right)\right|_{j R \rightarrow k R} ^{s}$ is the firstorder contribution of the time evolution operator which connects the initial state to a final state in the distant future, including only the parts of the tunnel Hamiltonian $H_{T}$ 
which describe tunneling of right-moving spin $s$ electrons from wire $j$ into wire $k$. In this way we can distinguish two events: an electron tunnels out of wire $1(1 \rightarrow 2)$, and an electron tunnels into wire $1(2 \rightarrow 1)$. One increases and the other decreases the current, but both add to the noise. Their strength is given by the overlap of the corresponding final state $U^{(1) s}|\downarrow \uparrow\rangle$ with its spin-flipped counterpart $\langle\uparrow \downarrow| U^{(1)-s^{\dagger}}$; i.e., a process has a large rate if the final state after one tunnel event is mostly invariant under spin flip. This will be a key observation to interpret the results. The factor $1+g / 2$ is caused by charge fractionalization [61]. When measuring the current or noise not in the TLL, but in Fermi liquid reservoirs to the right of the beam splitter, the complete charge will be detected [59,60, 62-66]. Formally, this corresponds to setting $g \rightarrow 1$ in the prefactors (but not in the correlation functions) of Eqs. (6) and (7). This, however, only leads to minor quantitative changes, so we will not make the distinction in the following.

In the noninteracting $(g=1)$ and in the symmetric $\left(x_{1}=x_{2}\right)$ cases, the time integrals in Eqs. (6) and (7) can be solved analytically. At $g=1$, the exchange noise is a Lorentzian of $d=x_{2}-x_{1}$,

$$
S_{12}^{(2) \operatorname{exc}}=e^{2} \frac{\Gamma_{2 e}}{2}\left|\frac{T}{\hbar v_{F}}\right|^{2} \frac{1}{4+(d / a)^{2}},
$$

meaning that there can only be an exchange process if the spins meet at the tunnel junction. Like in earlier noninteracting results in energy [31,34,35] and time domain [67], nonzero exchange noise requires orbital overlap. Interactions decrease the exchange signal. At $x_{1}=x_{2}$ the power law $S_{12}^{(2) \text { exc }} \propto((1+g) / 2)^{2}\left(2 g^{-1}+1\right)^{-\left(g^{-1}+g\right) / 2}$ is obtained.

As expected, the exchange current vanishes exactly without interactions, since the two amplitudes in Eq. (6) cancel. This is already true when spin-charge separation is neglected, i.e., when setting $v_{\rho}=v_{\sigma}$. For $v_{\rho} \neq v_{\sigma}$, however, a numerical integration of Eq. (6) demonstrates that $I_{1}^{(2) \text { exc }}$ is nonzero in general (Fig. 2). This confirms that entanglement can be detected in the many-body system by current measurements only and that the phenomenon of spincharge separation is essential. It induces a crucial asymmetry between the two competing processes, which goes unnoticed if both are summed up (current noise), but is relevant if they are subtracted (mean current).

The behavior of the exchange current in Fig. 2 can be qualitatively understood in the following way: In the nanowires, the two injected electrons decay each into a collective charge density excitation $\left\langle R \uparrow\left|\partial_{x} \theta_{i \rho}(x, t)\right| R \uparrow\right\rangle=$ $((1+g) / 2) \delta_{a}\left(x-x_{i}-v_{\rho}\left(t-t_{0}\right)\right)+((1-g) / 2) \delta_{a}(x-$ $\left.x_{i}+v_{\rho}\left(t-t_{0}\right)\right)$ and a collective spin density excitation $\left\langle R \uparrow\left|\partial_{x} \theta_{i \sigma}(x, t)\right| R \uparrow\right\rangle=\delta_{a}\left(x-x_{i}-v_{\sigma}\left(t-t_{0}\right)\right)$, where $\delta_{a}(x)=$ $(1 / \pi)\left(a /\left(a^{2}+x^{2}\right)\right)[11,59]$. They propagate with different

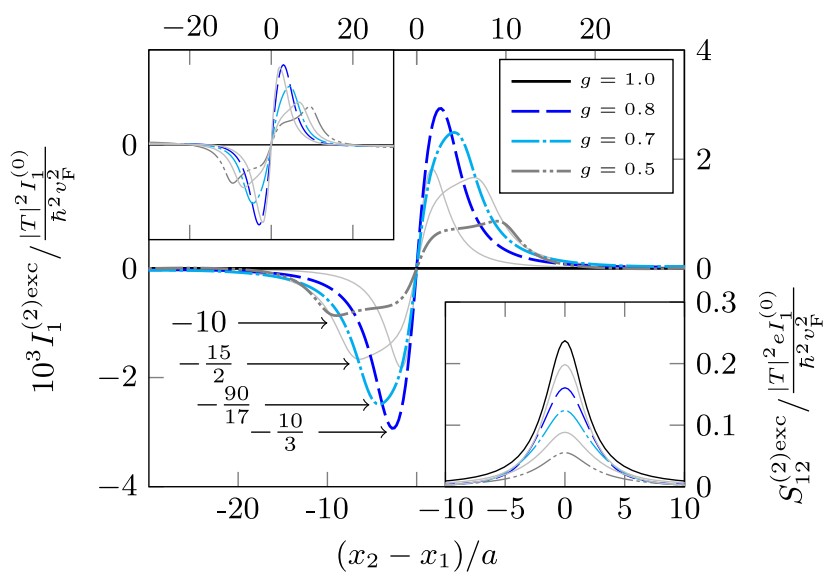

FIG. 2 (color online). Exchange contributions to the tunnel current in wire 1 and the zero-frequency current crosscorrelations between wire 1 and wire 2 (right inset) for different interaction parameters $g$ and injection distances. $\left(x_{1}+x_{2}\right) / 2=$ $-15 a$ is fixed. The exchange contribution to the tunnel current is nonzero if $x_{1} \approx x_{2}$ because spin-charge separation induces an asymmetry between the two directions of tunneling. The arrow tips indicate the expected positions of the maxima, cf. Eq. (9). Gray lines represent equidistant intermediate $g$ values. The exchange part of the current noise is finite only if the spins meet at the junction. Left inset: analytic approximation, Eq. (11).

velocities $v_{\rho, \sigma}$ and have a nonzero spatial extent $a$ due to the finite bandwidth. When one of them reaches the tunnel point at $x=0$, there is a charge or spin imbalance across the junction, which is compensated by a tunneling event: when the spin-down excitation in wire 1 arrives at the junction, either a spin-down electron can tunnel out of wire 1, or a spin-up electron can tunnel into wire 1 . So, quite intuitively, spin excitations alone do not create a charge current on average [68]. When, however, the charge excitation in wire 1 arrives at the tunnel contact, the charge imbalance induces only tunneling from wire 1 into wire 2 [first term in Eq. (6)]. It suffices to consider the case in which a spin-down electron tunnels [69]. As illustrated in Fig. 3(a), an additional charge excitation and an additional spin-down excitation are created in wire 2, and a spin-down hole is left behind in wire 1. This final state is invariant under spin flip if the two opposite spin excitations now present in each wire compensate. In wire 1, the spin hole must be compensated by the spin-down excitation from the injection. Because it is created at the position of the charge excitation, spin-charge separation makes this compensation impossible, unless the injection point in wire 1 is near the tunnel junction, so that both excitations are still close. In wire 2, the spin-down excitation produced by tunneling needs to coincide with the spin-up excitation created at injection, so the process is strong if $x_{1} / v_{\rho}=x_{2} / v_{\sigma}$. Following the same reasoning, the competing process $2 \rightarrow 1$ is strongest if $x_{2} / v_{\rho}=x_{1} / v_{\sigma}$. Unless $v_{\rho}=v_{\sigma}$ these conditions cannot be fulfilled simultaneously, so the two processes do not cancel and the 
(a)

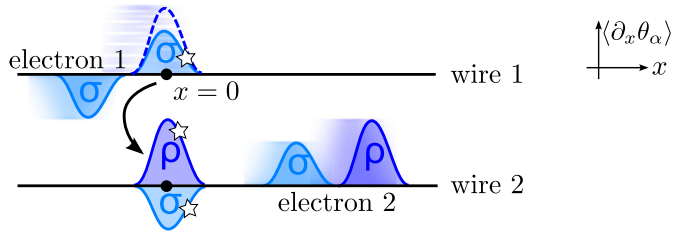

(b)

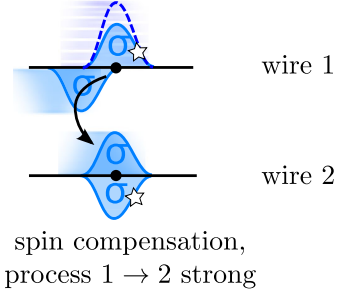

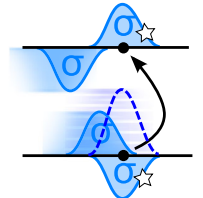

no spin compensation, process $2 \rightarrow 1$ weak

FIG. 3 (color online). Exchange process. (a) $x_{1} \ll x_{2}$. When the charge excitation of electron 1 (dashed line) reaches the tunnel junction at $x=0$, the charge imbalance can trigger a tunnel event. This creates a new charge excitation and a new spin excitation in wire 2 and leaves behind a spin hole in wire 1 (all marked by stars). Spin and charge excitations are drawn with different height for better visibility. (b) For suitable injection points $x_{1} / v_{\rho}=x_{2} / v_{\sigma}$ the new spin excitations compensate the one already present in each wire, leading to a strong exchange process. The competing process cannot have spin compensation at the same time and is weak. This asymmetry caused by spincharge separation gives rise to a finite exchange current.

exchange current becomes nonzero [Fig. 3(b)]. Using $g v_{\rho}=v_{\sigma}$, the two conditions can be combined as

$$
\frac{x_{2}-x_{1}}{x_{2}+x_{1}}= \pm \frac{g-1}{g+1}
$$

and become manifest as extrema in the exchange current (indicated by arrows in Fig. 2). Similar peaks reflecting spin-charge separation are already present in the direct terms (cf. the Supplemental Material [53]).

Quantitatively, the final state $1 \rightarrow 2$ is

$$
|1 \rightarrow 2\rangle:=\sum_{t} \psi_{2 \downarrow}^{\dagger}(0, t) \psi_{1 \downarrow}(0, t) \psi_{1 \downarrow}^{\dagger}\left(x_{1}, t_{0}\right) \psi_{2 \uparrow}^{\dagger}\left(x_{2}, t_{0}\right)|\rangle,
$$

where $t \in\left\{x_{1} / v_{\rho}, x_{1} / v_{\sigma}, x_{2} / v_{\sigma}\right\}$ is summed over all possible tunnel times (including the spin-induced events to allow for interference effects). The corresponding process strength is $P_{1 \rightarrow 2}:=\langle\widetilde{1 \rightarrow 2} \mid 1 \rightarrow 2\rangle$, where $|\widetilde{1 \rightarrow 2}\rangle$ is the spin-flipped final state, obtained by flipping all spin indices in Eq. (10). Constructing $P_{2 \rightarrow 1}$ analogously, the exchange current becomes

$$
I_{1}^{(2) \mathrm{exc}} \approx e \Gamma_{2 e}\left|\frac{T}{\hbar v_{\mathrm{F}}}\right|^{2} \frac{1+g}{2}\left(P_{2 \rightarrow 1}-P_{1 \rightarrow 2}\right) .
$$

All features of the numerics are reproduced by this expression (Fig. 2, left inset). From the explicit form of

$P_{i \rightarrow j}$, which is calculated in the Supplemental Material [53], we extract that the exchange current decays as $I^{(2) \operatorname{exc}} \propto\left|x_{1}+x_{2}\right|^{-3}$.

Biased injection.-Time controlled pointlike pair injection into QH edge states has recently been demonstrated with charge pumps [70]. A CPS operated at a constant voltage, on the other hand, can be modeled by a pairtunneling Hamiltonian which takes into account a voltage induced phase difference [71] instead of the initial state approach. The voltage gives rise to a length scale $\sim \hbar v_{F} / e V$. At large bias, $e V \rightarrow \hbar v_{F} a^{-1}$, the injection becomes as pointlike as allowed by the bandwidth and we recover the results of the initial state approach. At low voltages, it follows from standard renormalization group arguments that physical quantities like the current depend on the ratio of $x_{1,2}$ and the length scale set by the voltage [53]. In particular, the exchange currents at two voltages $V$, $V^{\prime}$ are related through

$$
I_{1 x_{1}, x_{2}}^{\mathrm{exc}}(V) \approx\left(\frac{V}{V^{\prime}}\right)^{g^{-1}+g-1} I_{1\left(V / V^{\prime}\right) x_{1},\left(V / V^{\prime}\right) x_{2}}^{\mathrm{exc}}\left(V^{\prime}\right) .
$$

This means that in order to access the $x_{1,2}$ dependency illustrated in Fig. 2 experimentally, it is not necessary to actually move the injection points. Rather, varying voltages can be applied to a fixed geometry sample (cf. the Supplemental Material [53]). In a QH realization, the length of different edges can be fine-tuned by appropriate gating, which additionally gives direct access to $x_{1,2}$.

To estimate the signal strength, we assume that the distance between the tunnel junction and the injection points is on the order of the length set by the bias voltage, $x_{1}=\hbar v_{F} / e V$ and $x_{2}=3 \hbar v_{F} / e V$. At smaller distances injection and tunneling cannot be regarded as distinct events. While not changing the physics, this considerably complicates quantitative predictions. At much larger distances, disorder and spin decoherence may become relevant. Employing the scaling relation [Eq. (12)], we obtain $I_{1 x_{1}, x_{2}}^{\text {exc }}(V) \approx e^{2} \hbar^{-1} V I_{1 x_{1}=a, x_{2}=3 a}^{\text {exc,init }} /\left(e v_{F} / a\right)$, where $I_{1}^{\text {exc,init }}$ is the exchange current obtained in the initial state approach. To remove the explicit dependency on the cutoff we have used $g^{-1}+g-1 \approx 1$, a valid approximation for a common nanowire interaction parameter $g=0.8$. A CPS is operated at voltages below the superconducting energy gap of about $1 \mathrm{meV}(\mathrm{Nb})$, such as $V \sim 0.1 \mathrm{mV}$. For a total transmission $|I|^{2}|T|^{2} \sim 10^{-2} \hbar^{4} v_{F}^{4}$, with $I$ the injection amplitude, the exchange current is on the order of a few $\mathrm{pA}$, a well-accessible value in experiments. With $v_{F}=10^{5} \mathrm{~m} / \mathrm{s}$, the injection distances become $\left|x_{1}\right| \sim 500 \mathrm{~nm}$ and $\left|x_{2}\right| \sim 1500 \mathrm{~nm}$.

The primary challenge when designing an entanglement detection scheme which is not based on the violation of a Bell-type inequality is to isolate the exchange contribution from the background given by the direct contributions and measurement noise. This is particularly true for the 
exchange current, which is 2 to 3 orders of magnitude smaller than the background. When changing the phase angle $\varphi$ linearly, the exchange contribution oscillates and can be isolated easily via lock-in amplification from the direct signal, which remains unaffected, cf. Eq. (3). All other parameters can then remain fixed. One way to influence $\varphi$ is given by the Rashba spin-orbit interaction [72] present in nanowires: when applying a transversal electric field $E$ (illustrated by the gray back gates in Fig. 1), spin-up and spin-down electrons acquire different Fermi vectors $k_{F} \pm k_{R}$ [32] where $k_{R}=2 \pi / \lambda_{R} \propto E$ is tunable via back gates [73,74]. In this way, until reaching the tunnel junction a relative phase $\varphi=4 \pi\left(x_{1}-x_{2}\right) / \lambda_{\mathrm{R}} \propto E$ is collected. Recent experiments on InAs wires show that the Rashba length $\lambda_{R}$ can become as short as $150 \mathrm{~nm}$ [75] which allows for several oscillation periods at the beam splitter size as estimated above. When ramping the electric field $E$ up and down in a triangular fashion, the exchange current oscillates continuously. Entanglement can thus be detected without any magnetic element or correlation measurement.

To conclude, we have demonstrated how, due to spincharge separation, the hallmark of TLLs, spin entanglement affects the average charge current in an electronic beam splitter. The underlying mechanism can be fully understood in terms of collective excitations. In addition to traditional entanglement detection schemes based on spin filters and correlation measurements, which have proven to be notoriously difficult to implement, this effect allows for a promising new approach.

We thank A. Baumgartner, F. Dolcini, T. Fujisawa, and B. Trauzettel for helpful discussions and comments. We acknowledge the support by the EU-FP7 project "Source of Entangled Electrons in Nano Devices" (SE2ND), No. 271554, the DFG Grant No. RE 2978/1-1 (P.R.), and Spanish MINECO through Grant No. FIS201126156 (A.L.Y.).

[1] M. A. Nielsen and I. L. Chuang, Quantum Computation and Quantum Information (Cambridge University Press, Cambridge, England, 2000).

[2] S. J. Freedman and J. F. Clauser, Phys. Rev. Lett. 28, 938 (1972).

[3] A. Aspect, J. Dalibard, and G. Roger, Phys. Rev. Lett. 49, 1804 (1982).

[4] G. Weihs, T. Jennewein, C. Simon, H. Weinfurter, and A. Zeilinger, Phys. Rev. Lett. 81, 5039 (1998).

[5] J. S. Bell, Physics 1, 195 (1964).

[6] J. F. Clauser, M. A. Horne, A. Shimony, and R. A. Holt, Phys. Rev. Lett. 23, 880 (1969).

[7] D. Loss and D. P. DiVincenzo, Phys. Rev. A 57, 120 (1998).

[8] R. Hanson, L. P. Kouwenhoven, J. R. Petta, S. Tarucha, and L. M. K. Vandersypen, Rev. Mod. Phys. 79, 1217 (2007).
[9] P. Recher, E. V. Sukhorukov, and D. Loss, Phys. Rev. B 63, 165314 (2001).

[10] G. Lesovik, T. Martin, and G. Blatter, Eur. Phys. J. B 24, 287 (2001).

[11] P. Recher and D. Loss, Phys. Rev. B 65, 165327 (2002).

[12] C. Bena, S. Vishveshwara, L. Balents, and M. P. A. Fisher, Phys. Rev. Lett. 89, 037901 (2002).

[13] P. Recher and D. Loss, Phys. Rev. Lett. 91, 267003 (2003).

[14] A. Levy Yeyati, F. S. Bergeret, A. Martin-Rodero, and T. M. Klapwijk, Nat. Phys. 3, 455 (2007).

[15] J. Cayssol, Phys. Rev. Lett. 100, 147001 (2008).

[16] K. Sato, D. Loss, and Y. Tserkovnyak, Phys. Rev. Lett. 105, 226401 (2010).

[17] J. Torrès and T. Martin, Eur. Phys. J. B 12, 319 (1999).

[18] G. Falci, D. Feinberg, and F. W. J. Hekking, Europhys. Lett. 54, 255 (2001).

[19] L. Hofstetter, S. Csonka, J. Nygård, and C. Schönenberger, Nature (London) 461, 960 (2009).

[20] L. G. Herrmann, F. Portier, P. Roche, A. L. Yeyati, T. Kontos, and C. Strunk, Phys. Rev. Lett. 104, 026801 (2010).

[21] A. Das, Y. Ronen, M. Heiblum, D. Mahalu, A. V. Kretinin, and H. Shtrikman, Nat. Commun. 3, 1165 (2012).

[22] J. Schindele, A. Baumgartner, and C. Schönenberger, Phys. Rev. Lett. 109, 157002 (2012).

[23] S. Kawabata, J. Phys. Soc. Jpn. 70, 1210 (2001).

[24] N. M. Chtchelkatchev, G. Blatter, G. B. Lesovik, and T. Martin, Phys. Rev. B 66, 161320 (2002).

[25] P. Samuelsson, E. V. Sukhorukov, and M. Büttiker, Phys. Rev. Lett. 91, 157002 (2003).

[26] C. W. J. Beenakker, C. Emary, M. Kindermann, and J. L. van Velsen, Phys. Rev. Lett. 91, 147901 (2003).

[27] P. Samuelsson, E. V. Sukhorukov, and M. Büttiker, Phys. Rev. Lett. 92, 026805 (2004).

[28] O. Sauret, T. Martin, and D. Feinberg, Phys. Rev. B 72, 024544 (2005).

[29] W. Chen, R. Shen, L. Sheng, B. G. Wang, and D. Y. Xing, Phys. Rev. Lett. 109, 036802 (2012).

[30] B. Braunecker, P. Burset, and A. Levy Yeyati, Phys. Rev. Lett. 111, 136806 (2013).

[31] G. Burkard, D. Loss, and E. V. Sukhorukov, Phys. Rev. B 61, R16303 (2000).

[32] J. C. Egues, G. Burkard, and D. Loss, Phys. Rev. Lett. 89, 176401 (2002).

[33] G. Burkard and D. Loss, Phys. Rev. Lett. 91, 087903 (2003).

[34] X. Hu and S. Das Sarma, Phys. Rev. B 69, 115312 (2004).

[35] P. Samuelsson, E. V. Sukhorukov, and M. Büttiker, Phys. Rev. B 70, 115330 (2004).

[36] J. C. Egues, G. Burkard, D. S. Saraga, J. Schliemann, and D. Loss, Phys. Rev. B 72, 235326 (2005).

[37] V. Giovannetti, D. Frustaglia, F. Taddei, and R. Fazio, Phys. Rev. B 74, 115315 (2006).

[38] P. San-Jose and E. Prada, Phys. Rev. B 74, 045305 (2006).

[39] F. Mazza, B. Braunecker, P. Recher, and A. Levy Yeyati, Phys. Rev. B 88, 195403 (2013).

[40] A. Komnik and R. Egger, Phys. Rev. Lett. 80, 2881 (1998).

[41] A. Komnik and R. Egger, Eur. Phys. J. B 19, 271 (2001).

[42] Y. Ji, Y. Chung, D. Sprinzak, M. Heiblum, D. Mahalu, and H. Shtrikman, Nature (London) 422, 415 (2003). 
[43] I. Neder, M. Heiblum, Y. Levinson, D. Mahalu, and V. Umansky, Phys. Rev. Lett. 96, 016804 (2006).

[44] I. Neder, N. Ofek, Y. Chung, M. Heiblum, D. Mahalu, and V. Umansky, Nature (London) 448, 333 (2007).

[45] E. Bocquillon, V. Freulon, J.-M. Berroir, P. Degiovanni, B. Plaçais, A. Cavanna, Y. Jin, and G. Fève, Science 339, 1054 (2013).

[46] C. Wahl, J. Rech, T. Jonckheere, and T. Martin, Phys. Rev. Lett. 112, 046802 (2014).

[47] S.-i. Tomonaga, Prog. Theor. Phys. 5, 544 (1950).

[48] J. M. Luttinger, J. Math. Phys. (N.Y.) 4, 1154 (1963).

[49] J. Kim, K. Kang, J.-O. Lee, K.-H. Yoo, J.-R. Kim, J. W. Park, H. M. So, and J.-J. Kim, J. Phys. Soc. Jpn. 70, 1464 (2001).

[50] B. Gao, A. Komnik, R. Egger, D. C. Glattli, and A. Bachtold, Phys. Rev. Lett. 92, 216804 (2004).

[51] B. Karmakar, D. Venturelli, L. Chirolli, F. Taddei, V. Giovannetti, R. Fazio, S. Roddaro, G. Biasiol, L. Sorba, V. Pellegrini, and F. Beltram, Phys. Rev. Lett. 107, 236804 (2011).

[52] E. Bocquillon, V. Freulon, J.-M. Berroir, P. Degiovanni, B. Plaçais, A. Cavanna, Y. Jin, and G. Fève, Nat. Commun. 4, 1839 (2013).

[53] See Supplemental Material at http://link.aps.org/ supplemental/10.1103/PhysRevLett.113.266401 for further details.

[54] J. von Delft and H. Schoeller, Ann. Phys. (Berlin) 7, 225 (1998).

[55] H. Grabert, in Exotic States in Quantum Nanostructures, edited by S. Sarkar (Kluwer Academic, Dordrecht, 2002), Chap. 1.

[56] T. Giamarchi, Quantum Physics in One Dimension (Oxford University Press, New York, 2003).

[57] In a spinless TLL at sufficiently strong interactions, $g<1 / 2$, electrostatic interwire effects become relevant (in the renormalization group sense), which change the model substantially [40]. Additional noninteracting channels like spin lower this bound [50], but we shall conservatively limit this discussion to moderate interaction strengths $1 / 2<g \leq 1$.

[58] C. L. Kane and M. P. A. Fisher, Phys. Rev. Lett. 72, 724 (1994).

[59] A. Crépieux, R. Guyon, P. Devillard, and T. Martin, Phys. Rev. B 67, 205408 (2003).

[60] F. Dolcini, B. Trauzettel, I. Safi, and H. Grabert, Phys. Rev. B 71, 165309 (2005).

[61] K.-V. Pham, M. Gabay, and P. Lederer, Phys. Rev. B 61, 16397 (2000).

[62] D. L. Maslov and M. Stone, Phys. Rev. B 52, R5539 (1995).

[63] V. V. Ponomarenko, Phys. Rev. B 52, R8666 (1995).

[64] I. Safi and H. J. Schulz, Phys. Rev. B 52, R17040 (1995).

[65] A. V. Lebedev, A. Crépieux, and T. Martin, Phys. Rev. B 71, 075416 (2005).

[66] P. Recher, N. Y. Kim, and Y. Yamamoto, Phys. Rev. B 74, 235438 (2006).

[67] S. Ol'khovskaya, J. Splettstoesser, M. Moskalets, and M. Büttiker, Phys. Rev. Lett. 101, 166802 (2008).

[68] Spin excitations do, however, create charge noise.

[69] If a spin-up electron tunnels, there cannot be any overlap with the spin-flipped state, as the total spin per wire does not vanish. The event is explicitly ruled out by Klein factors in Eq. (6).

[70] N. Ubbelohde, F. Hohls, V. Kashcheyevs, T. Wagner, L. Fricke, B. Kästner, K. Pierz, H. W. Schumacher, and R. J. Haug, Nat. Nanotechnol. (in press).

[71] G. D. Mahan, Many-Particle Physics (Kluwer Academic, New York, 1981).

[72] Y. A. Bychkov and E. I. Rashba, J. Phys. C 17, 6039 (1984).

[73] D. Liang and X. P. Gao, Nano Lett. 12, 3263 (2012).

[74] Y. Kanai, R. S. Deacon, S. Takahashi, A. Oiwa, K. Yoshida, K. Shibata, K. Hirakawa, Y. Tokura, and S. Tarucha, Nat. Nanotechnol. 6, 511 (2011).

[75] P. Wójcik, J. Adamowski, B. J. Spisak, and M. Wołoszyn, J. Appl. Phys. 115, 104310 (2014). 